\title{
Therapeutic Cycles Model (TCM): instrumento de avaliação do processo psicoterapêutico
}

\author{
Paula Argemi Cassel - Pontifícia Universidade Católica do Rio Grande do Sul, Porto Alegre, Brasil \\ Livia Fração Sanchez - Pontifícia Universidade Católica do Rio Grande do Sul, Porto Alegre, Brasil \\ Paula von Megden Campezatto - Pontifícia Universidade Católica do Rio Grande do Sul, Porto Alegre, Brasil \\ Instituo de Ensino e Pesquisa em Psicoterapia, Porto Alegre, Brasil \\ Eduardo Khater - Pontificia Universidade Católica de Campinas, Campinas, Brasil \\ Maria Lúcia Tiellet Nunes - Pontifícia Universidade Católica do Rio Grande do Sul, Porto Alegre, Brasil \\ Elisa Yoshida - Pontifícia Universidade Católica de Campinas, Campinas, Brasil
}

\begin{abstract}
Resumo
O objetivo deste trabalho foi apresentar a versão brasileira do Therapeutic Cycles Model (TCM) a partir da análise de uma sessão de um processo de psicoterapia psicanalítica. O TCM é um método de análise de textos por computador que identifica os momentos-chave no discurso de paciente, psicoterapeuta, ou de ambos, em interação. As análises são feitas com o auxílio de um software (Cycles Model), que dispõe de listas de palavras de tom emocional e de abstrações, permitindo a classificação do texto em duas categorias: conteúdo emocional e cognitivo das verbalizações na sessão. Buscou-se ilustrar uma possibilidade de utilização do TCM a partir de uma sessão de psicoterapia. A análise exploratória dos conteúdos verbalizados pela dupla, paciente e psicoterapeuta, a partir dos ciclos terapêuticos demonstrou que esse instrumento contribui na identificação de momentos clinicamente significativos do discurso do paciente em sessões individuais. Sendo assim, o TCM pode ser mais uma ferramenta para compreender o processo terapêutico e, com isso, auxiliar o psicoterapeuta em sua prática.

Palavras-chave: psicoterapia; avaliação de processo; pesquisa empírica.
\end{abstract}

\section{Therapeutic Cycles Model (TCM): instrument of evalution of psychotherapeutic process}

\begin{abstract}
The objective of this study was to present the Brazilian version of Therapeutic Cycles Model (TCM) from the analysis of a psychoanalytic psychotherapeutical process. TCM is a method of computer analysis of texts by identifying key-moments in the speech of the patient, therapist, or both in interaction. The analysis is performed with the software (Cycles Model), which provides word lists of emotional tone and abstractions, allowing classification of text into two categories: emotional and cognitive content of utterances in the session. We have tried to illustrate a possibility of TCM's use from a psychotherapy session. Exploratory analysis of the contents verbalized by the double patient and psychotherapist demonstrated that this instrument helps in identifying clinically significant moments in speech of the patient in individual sessions. Thus, the TCM can be a tool in comprehending psychotherapeutic processes, helping psychotherapist in practice.

Keyworks: psychotherapy; review process; empirical research.
\end{abstract}

Therapeutic Cycles Model (TCM): instrumento de evaluación del proceso psicoterapéutico

\begin{abstract}
Resumen
El objetivo de este trabajo fue presentar la versión brasileña del Therapentic Cycles Model (TCM) partiendo del análisis de una sesión de un proceso de psicoterapia psicoanalítica. El TCM es un método computarizado de análisis de textos que identifica los momentos clave en el discurso del paciente, del psicoterapeuta o de la interacción entre ambos. El proceso de análisis se realiza con el auxilio de un software (Cycles Model), que dispone de listas de palabras de tono emocional y de abstracciones, permitiendo la clasificación del texto en dos categorías: contenido emocional y contenido cognitivo de las verbalizaciones durante la sesión. Se buscó la posibilidad de utilización del TCM a partir de una sesión de psicoterapia. El análisis exploratorio de los contenidos verbalizados por la dupla paciente/psicoterapeuta demostró que este instrumento ayuda en la identificación de momentos clínicamente significativos del discurso del paciente en sesiones individuales. De este modo, el TCM puede ser una herramienta más para comprender el proceso terapéutico y por lo tanto auxiliar al psicoterapeuta en su práctica.

Palabras-clave: psicoterapia; evaluación del procesos; investigación empírica.
\end{abstract}

\section{Introdução}

Atualmente, as tendências de pesquisas em psicoterapia e psicoterapia psicanalítica apontam para o estudo do processo psicoterapêutico por meio de métodos empíricos que objetivam analisar o próprio processo, bem como os padrões de comunicação entre a dupla paciente-psicoterapeuta (Charman, 2003; Eizirik, 2006). As pesquisas empíricas, nessa modalidade, possibilitam respostas mais específicas a respeito da psicoterapia nos diferentes tipos de tratamento (Charman, 2003; Fishman, 2002; Kächele, 2000), uma vez 
que o estudo do processo psicoterapêutico permite ir ao encontro de como se dão as mudanças no decorrer do tratamento psicoterapêutico (Brum et al., 2012).

Bucci (2007) caracteriza a pesquisa de processo psicoterapêutico como o método particular de investigação sistemática da experiência humana em contexto interpessoal. Nesse sentido, seu principal objetivo é identificar e validar mudanças nos padrões emocionais não adaptativos do paciente. Isto é, na avaliação do processo de psicoterapia, é relevante estudar momentos passíveis de identificação de mudanças no paciente.

O estudo do processo psicoterapêutico pode ser realizado por meio da análise minuciosa das interações psicoterapêuticas, o que foi facilitado pelo uso de tecnologias, como de gravação em áudio e filmagem das sessões. A inclusão de registros objetivos das sessões (Bucci, 2007; Greenberg \& Newman, 1996; Serralta \& Streb, 2003) ainda são pouco aceitas pela comunidade psi (Meyer, 1988). Psicoterapeutas clínicos costumam temer a utilização desse instrumental pelo receio da exposição do paciente e de interferência negativa no processo psicoterapêutico, bem como no desenvolvimento da aliança terapêutica (Silva, Halberg, Steibel, \& Campezzato, 2010). Kächele, Thomä, Ruberg, \& Grünzig (1988) referem que uma parcela de psicanalistas afirma que o gravador exerce o papel de uma terceira pessoa no setting terapêutico.

Essas resistências dificultam a realização de pesquisas empíricas em psicoterapia psicanalítica e o resultado disso é o escasso número de publicações na área (Ferreira \& Yoshida, 2004; Nunes, 1995). Tal aspecto é negativo, em especial para a clínica, porque a tendência atual é privilegiar a medicina baseada em evidências (Deakin \& Nunes, 2008). Com o aparecimento dos planos de saúde privados e da assistência pública, a procura por evidências tornou-se relevante na escolha de terapêuticas, e a inclusão de método de pesquisa que não faziam parte do método psicanalítico ou da psicoterapia psicanalítica tornou-se necessária.

Uma das formas de estudar o processo psicoterapêutico é por meio de estudos de casos, especialmente os de caso único (Serralta, Nunes, \& Eizirik, 2011). Esse método é considerado imprescindível para produção de conhecimento em psicoterapia (Bucci, 2007), pois, a partirdele, pode ser possível atenuar a distância entre prática clínica e pesquisa empírica (Serralta, Nunes, \& Eizirik, 2007). Nos estudos de caso, é possível o uso de instrumentos nas pesquisas de processo, pois almejam medir variáveis do processo psicoterapêutico em sessões transcritas ou gravadas em áudio e/ou vídeo (Almeida, 2010). Os dados coletados podem ser examinados pelo psicoterapeuta, bem como por juízes independentes (Peuker, Habigzang, Koller, \& Araújo, 2009). No Brasil, percebe-se o aumento do interesse dos pesquisadores em elaborarem versões brasileiras de instrumentos que avaliem fatores que fazem parte do processo psicoterapêutico, uma vez que há poucos instrumentos disponíveis aos pesquisadores brasileiros para análise deste (Serralta et al., 2007). Exemplos desses instrumentos, no Brasil, são: o Psychotherapy Process Q-Set (PQS), adaptado para a versão em português por Serralta, Nunes e Eizirik (2007); a Aliança Psicoterápica da Califórnia, na versão do paciente (Calpas-P), adaptada para o Brasil por Marcolino e Iacoponi (2001); o Instrumento de Avaliação de Sessões Psicanalíticas (IASP), elaborado por Almeida (Almeida, 2010; Hauck, Crestana, Mombach, Almeida, \& Eizirik, 2008); o desenvolvimento da versão em português do Brasil do questionário sobre padrão de relacionamentos Beziehungs-Muster Fragebongen (Ferreira et al., 2006) e a adaptação da versão brasileira do Therapentic Cycles Model - Modelo dos Ciclos Terapêuticos, apresentado no presente artigo.

Sendo assim, o objetivo deste trabalho é apresentar a versão brasileira do instrumento denominado Therapentic Cycles Model (TCM), cujo objetivo é identificar momentos-chave de um processo psicoterapêutico. Inicialmente desenvolvido por Erhard Mergenthaler (1996a), na Alemanha, foi adaptado para o Brasil por Elisa Yoshida (2008). Atualmente, estão sendo realizadas pesquisas no Rio Grande do Sul para adaptação do instrumento para a realidade brasileira (Campezatto, 2014; Cassel, 2014; Sanchez, 2014). A ilustração do uso do TCM será feita a partir de um caso clínico de psicoterapia psicanalítica.

\section{Therapeutic Cycles Model - Modelo dos Ciclos Terapêuticos}

O TCM foi desenvolvido para identificar momentos de mudança em psicoterapia. É um método de análise de textos que, quando aplicado a transcrições de sessões de psicoterapia, permite a identificação de momentos-chave do processo psicoterapêutico, nomeados ciclos terapêuticos. Um momento-chave se refere "a uma ou mais sessões de um tratamento ou a um segmento de sessão visto como clinicamente importantes" (Mergenthaler, 1996b, p. 1306) e podem ser encontrados por meio da narrativa do paciente, do psicoterapeuta ou de ambos quando em interação. São 
momentos nos quais é possível observar mudanças no paciente e que se relacionam à noção de progresso clínico (Mergenthaler, 1996b).

A demarcação dos momentos-chave é realizada por meio da identificação pelo software de marcadores verbais que comportam a expressão de emoções positivas ou negativas e de abstrações positivas ou negativas. Os marcadores emocionais são palavras que pertencem a uma ou mais das seguintes dimensões: prazer-desprazer, aprovação-desaprovação, apego-desapego, e surpresa. Segundo Yoshida (2008), o TCM fundamenta-se no entendimento de que a expressão de emoções é fator central de todo e qualquer processo psicoterapêutico. Contudo, deve estar associada à capacidade do sujeito em tratamento perceber $\mathrm{e}$ refletir sobre sua experiência vivida e de expressá-la verbalmente; isto é, o modelo de análise de mudança do TCM parte do pressuposto que o processo de mudança terapêutica é conduzido pela interação entre experiência emocional e cognitiva tanto do paciente como do psicoterapeuta.

\section{Avaliação empírica do TCM}

O Modelo dos Ciclos Terapêuticos é operacionalizado a partir do programa de análise de dados por computador CM (Cycles Model) que mede a presença de palavras de transcrições de sessões psicoterapêuticas. Isso ocorre a partir de um dicionário desenvolvido previamente e que pode ser adaptado ou customizado de forma individual, ou seja, incluir palavras não contempladas inicialmente. As palavras pertencentes ao dicionário são categorizadas de acordo com a natureza do tom emocional (positiva ou negativa) ou da indicação de uma abstração. Neste último caso, são divididas em abstrações ou abstrações com conotação afetiva positiva e negativa (Mergenthaler, 1996b). Exemplos de palavras com tom emocional positivo seriam: "abraçar, ajudar". Com tom emocional negativo: "rejeitar, frustrar". Dentre as abstrações, "departamento, ano, febre, medicina". Abstração com tom positivo: "amizade, amor". E, por fim, abstrações de tom negativo: "humilhação, injustiça". Os dicionários são desenvolvidos a partir de inúmeros textos que comprovam que os marcadores são significativos ao indicar abstração e tom emocional na linguagem (Mergenthaler, 1996b).

\section{Dicionário de palavras emocionais}

As palavras de tom emocional são classificadas como positivas (1) ou negativas (2), de acordo com os critérios:
- Sentimentos de prazer/desprazer: Se o sentimento estiver relacionado a prazer, a palavra é classificada como positiva (1) (ex.: aproveitando); se o sentimento é ligado ao desprazer, a palavra é classificada como negativa (2) (ex.: tedioso) (Khater \& Yoshida, 2011).

- Avaliação cognitiva: aprovação/desaprovação: Se a avaliação é relacionada à aprovação, a palavra deve ser classificada como positiva (1) (ex.: aceitar); se a avaliação é relacionada à desaprovação, a palavra deve ser classificada como negativa (2) (ex.: desprezar) (Khater \& Yoshida, 2011).

- Relacionamento interpessoal: apego/desapego: Se o valor emocional for relacionado a apego, a palavra deve ser classificada como positiva (1) (ex.: amar); se o valor emocional for relacionado a desapego, a palavra deve ser classificada como negativa (2) (ex.: sozinho) (Khater \& Yoshida, 2011).

- Surpresa: Se a palavra expressa sentimento de surpresa, deve ser classificada como positiva (1) (ex.: surpreso) (Khater \& Yoshida, 2011).

\section{Dicionários de palavras que conotam abstração}

Os substantivos que referem a um conceito abstrato sem valor emocional foram classificados como abstração (3) (ex.: plano, teste, palavra, drogas) (Khater \& Yoshida, 2011).

No caso em que os substantivos são abstratos e contêm tom emocional, eles são classificados de acordo com os mesmos critérios das palavras de tom emocional:

- Sentimentos de prazer/desprazer: Se o sentimento for relacionado a prazer, a palavra deve ser classificada como abstração com tom emocional positivo (4) (ex.: entusiasmo); se o sentimento for relacionado ao desprazer, a palavra deve ser classificada como abstração com tom emocional negativo (5) (ex.: tristeza) (Khater \& Yoshida, 2011).

- - Avaliação cognitiva: aprovação/desaprovação: Se a palavra remete a uma avaliação cognitiva relacionada à aprovação, ela deve ser classificada como abstração e tom emocional positivo (4) (ex.: apego); se a avaliação é relacionada à desaprovação, a palavra deve ser classificada como abstração e tom emocional negativo (5) (ex.: solidão) (Khater \& Yoshida, 2011).

- Relacionamento interpessoal: apego/desapego: Se o valor emocional for relacionado a apego, a 
palavra deve ser classificada como tom emocional positivo (4) (ex.: fé); se o valor emocional for relacionado a desapego, a palavra deve ser classificada como tom emocional negativo (5) (ex.: solidão) (Khater \& Yoshida, 2011).

Os dicionários customizados são complementados a partir de cada estudo realizado e anexados no software $\mathrm{CM}$ que analisa as transcrições de sessões quanto à ocorrência de palavras ligadas à emoção e à abstração. A análise do texto pelo softwware fornece a frequência de palavras integradas a cada uma das categorias, permitindo a avaliação qualitativa que se traduz em padrões de emoção-abstração. $O$ texto é usualmente fragmentado em blocos de palavras, os quais devem ser suficientes para possibilitar a identificação da ocorrência dos padrões de comunicação em cada bloco e ao longo da sessão como um todo. A decisão do número de palavras de cada bloco fica a critério do usuário, mas tem, em geral, entre 100 a 200 palavras, sendo o mais usual de 150 palavras. Uma vez definido o tamanho do bloco, ele será constante para todo o texto a ser analisado. Se o tamanho do último bloco é menor que o comprimento definido, este se junta ao bloco anterior. O software avalia esses blocos de palavras conforme o tom emocional (TE) e uso de abstrações $(\mathrm{AB})$, bem como gera gráficos representativos dos padrões predominantes de cada bloco ou do processo psicoterapêutico como um todo, este último denominado corpus overview (Mergenthaler, 1996b).

A partir da análise dos gráficos, é possível determinar padrões clinicamente relevantes durante processos de psicoterapias, com base na verbalização do paciente, do terapeuta e de ambos em interação. Eles são dados a partir das frequências de palavras identificadas como de tom emocional ou de abstração. Mergenthaler (1996b) descreveu quatro padrões: padrão A - relaxamento (relaxing); padrão B - reflexão (reflecting); padrão $\mathrm{C}$ - experiência (experiencing) e padrão D - conexão (connecting), os quais se caracterizam da seguinte forma:

- Padrão A - relaxamento: baixo tom emocional e baixa abstração.

Os pacientes falam sobre um material que não está manifestamente conectado aos seus sintomas ou temas centrais (Mergenthaler, 1996b). É um momento de descrição da rotina diária, de situações, sem a inclusão de processos reflexivos sobre a descrição. Além disto, é um estado para o qual os pacientes retornam sempre que sentem necessidade, dessa forma, regeneram tanto o físico quanto a psique. Nesse momento, geralmente, aparecem relatos de conteúdos irrelevantes na terapia. Essa é uma fase de caráter genérico que representa um descanso, uma pausa durante o curso da sessão.

- Padrão B - reflexão: baixo tom emocional e alta abstração.

Os pacientes apresentam tópicos com alto grau de abstração e sem emoções associadas (Mergenthaler, 1996b). Isso pode ser a expressão da defesa conhecida como intelectualização.

- Padrão C - experiência: alta emoção e baixa abstração.

Os pacientes se encontram num estado de experiência emocional. Eles podem estar abordando temas conflituosos ao mesmo passo em que os vivenciam emocionalmente. O paciente está experimentando as emoções ligadas a experiências passadas, mas sem elaboração cognitiva destas (Mergenthaler, 1996b).

- Padrão D - conexão: alto tom emocional e alta abstração.

Os pacientes encontraram acesso emocional para o tema conflituoso e refletem sobre eles. Esse estado marca um momento clinicamente importante (insight). Esse é um instante que foi denominado momento-chave (Mergenthaler, 1996b).

A Figura 1 oferece uma representação gráfica de cada um desses padrões, e a Figura 2 fornece uma representação gráfica sintética de como os padrões são compostos.

A partir da identificação dos padrões verbalizados pelo paciente, pelo terapeuta, ou ambos, é possível observar a ocorrência de ciclos terapêuticos, ou seja, uma "sequência temporal específica dos quatro padrões de emoção abstração (Mergenthaler, 1996b, p. 1308)". Cada padrão (relaxamento, reflexão, experiência e conexão) corresponde a uma fase do ciclo terapêutico, como descrito a seguir (Mergenthaler, 2008):

Fase I - Inicia-se com o padrão A (relaxamento) e corresponde aos momentos em que a pessoa descreve episódios de sua vida, ou se refere a temas com baixa conotação afetiva. A narrativa pode revelar tanto um estado de relaxamento quanto de desorientação, em que a pessoa parece não saber ao certo sobre o que falar 


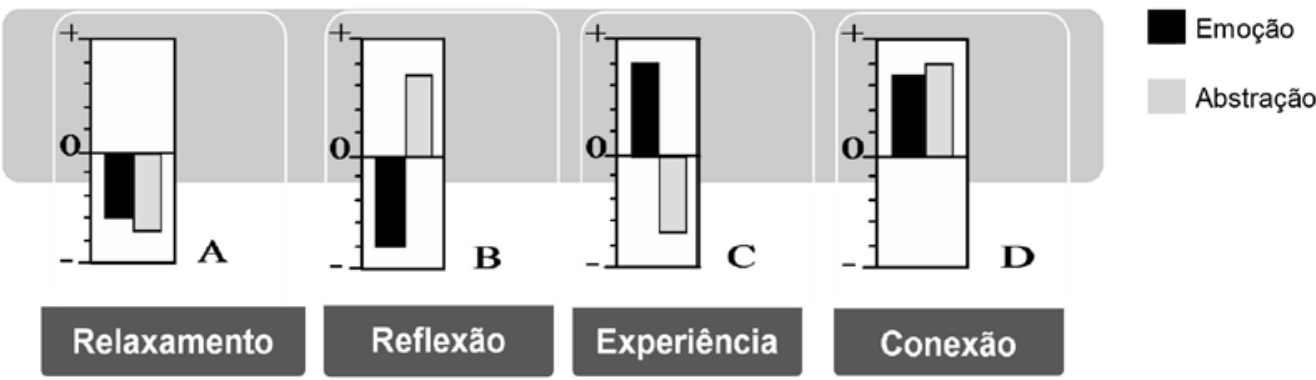

Figura 1. Esquema gráfico dos padrões de abstração/emoção (Mergenthaler, 1996b; Yoshida \& Mergenthaler, 2011).

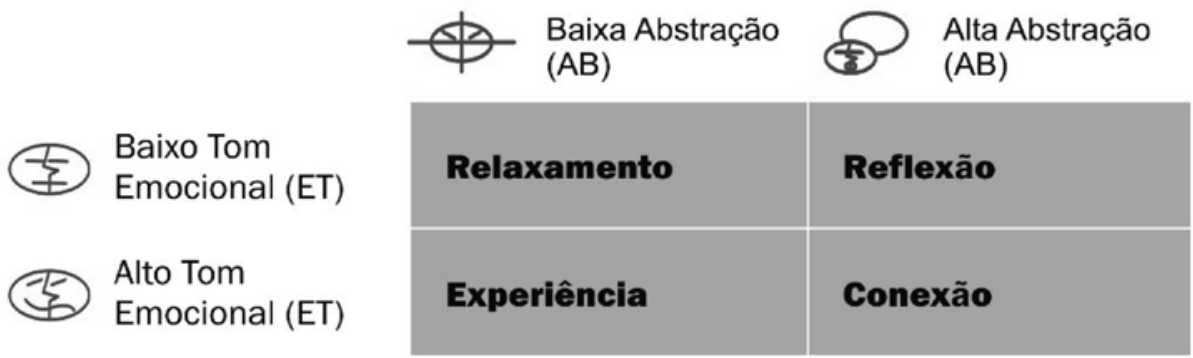

Figura 2. Representação dos padrões de abstração/emoção (fonte: autores).

(Buchheim \& Mergenthaler, 2002). Prevalecem palavras com baixo tom emocional (TE) e baixa abstração (AB) (Mergenthaler, 1996b);

Fase II - Na medida em que a pessoa começa a falar de seus sintomas e de suas dificuldades, aumenta a chance de verbalização com conteúdo emocional e, portanto, do aparecimento do padrão C (experiência). A pessoa se refere a situações difíceis com forte conotação afetiva, sem ainda poder elaborá-las cognitivamente. Prevalece o uso de palavras com alto TE e baixa AB (Mergenthaler, 1996b);

Fase III - É marcada pela associação de expressão verbal das emoções, acompanhada da possibilidade de refletir sobre elas. O que caracteriza, portanto, o padrão $\mathrm{D}$ (conexão). Teoricamente, essa esta fase pode refletir um momento de elaboração e estar associada a um insight (Buchheim \& Mergenthaler, 2002). Observam-se palavras com alto TE e alta AB (Mergenthaler, 1996b).

Fase IV - Como consequência do entendimento e da nova compreensão favorecidos pelo insight, um momento de reflexão sobre a experiência vivida costuma ocorrer, marcando, portanto, uma fase caracterizada pela ocorrência do padrão B (reflexão) que se traduz por alta AB e baixo TE (Mergenthaler, 1996b);
Fase $\mathrm{V}$ - A quinta fase marca o final do ciclo, com o retorno a um estado de relaxamento (padrão A) e a preparação para um novo ciclo e, portanto, baixo TE e baixa AB (Mergenthaler, 1996b). A Figura 3 apresenta a representação gráfica desse modelo.

Após revisão da literatura do TCM, exemplifica-se sua utilização na avaliação de mudança em processos psicoterapêuticos individuais (Lepper \& Mergenthaler, 2005) e em grupo (Fontao \& Mergenthaler, 2002). A aplicação do software admitiu a identificação de momentos-chave nos processos psicoterapêuticos, estando associado, por exemplo, a situações de insight (Mergenthaler \& Bucci, 1999) ou silêncio durante as sessões psicoterapêuticas (Levitt, Butler, \& Hill, 2005). Esse instrumento também pode ser usado em estudos sobre análise das ferramentas de linguagem do terapeuta e do paciente (Gelo \& Mergenthaler, 2003) e na identificação de momentos-chave em grupo de adolescentes com linguagens específicas (Khater \& Yoshida, 2011).

A partir da explanação acerca do TCM, busca-se apresentar sua utilização a partir de um exemplo de um caso clínico - uma sessão de um processo de psicoterapia psicanalítica. 


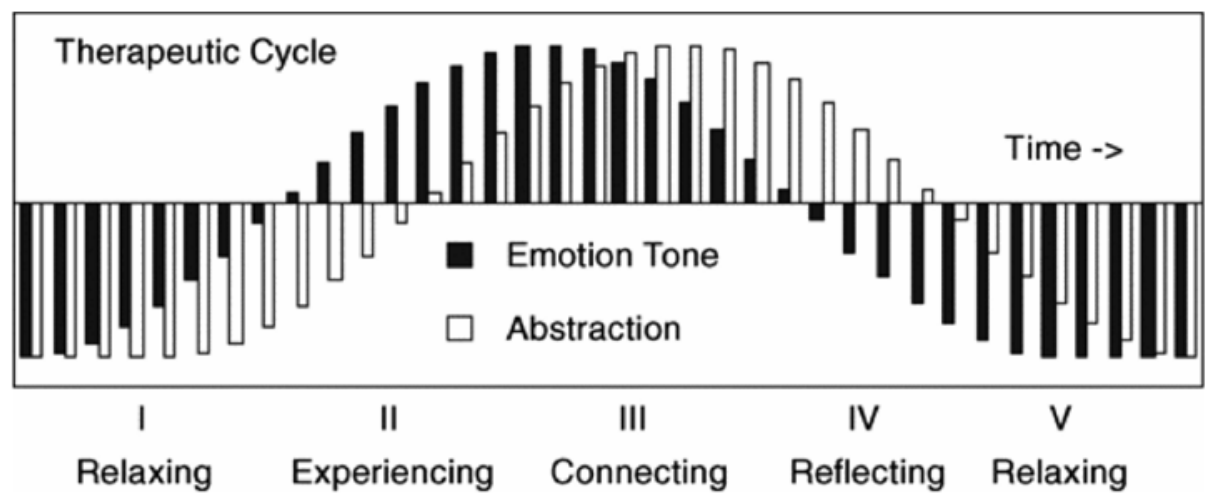

Figura 3. Modelos de ciclos terapêuticos: fluxo prototípico acima e abaixo da média de tons de emoção e abstração ao longo do tempo (Mergenthaler, 1996b).

\section{Método}

\section{Delineamento}

Estudo de abordagem qualitativa e descritiva, com delineamento de estudo de caso único sistemático (Edwards, 2007).

\section{Participantes}

Fez parte do estudo uma dupla paciente-psicoterapeuta $^{1}$. A paciente Maria ${ }^{2}$ é adulta e buscou atendimento psicológico em um serviço de atendimento psicoterapêutico de Porto Alegre - RS e realizou processo de triagem, a fim de ser avaliada para indicação de psicoterapia psicanalítica e exclusão de necessidade de internação psiquiátrica.

\section{Procedimentos de coleta e análise de dados}

A coleta de dados (gravação em áudio das sessões de psicoterapia psicanalítica) foi realizada em um serviço de atendimento psicoterapêutico de Porto Alegre - RS, bem como a transcrição literal do material gravado. De acordo com os procedimentos éticos da pesquisa, a paciente foi convidada e aceitou participar

\footnotetext{
${ }^{1}$ Os dados apresentados, neste artigo, foram cedidos da pesquisa " $\mathrm{A}$ resistência no início do tratamento - estudo do processo da psicoterapia" (Silva et al., 2010). Os procedimentos éticos foram atendidos - aprovação da pesquisa pelo Comitê de Ética em Pesquisa da Pontifícia Universidade Católica do Rio Grande do Sul (parecer $\mathrm{n}^{\circ}$.: 487.284, CAAE: 15575613.4.0000.5336).

${ }^{2}$ Codinome escolhido por ser de uso comum no Brasil e por representar uma mulher pura, forte, com virtudes, requisitos esses pertences à paciente.
}

da pesquisa mediante assinatura do Termo de Consentimento Livre e Esclarecido.

Esta pesquisa foi realizada a partir da leitura das transcrições de todas as sessões e foi escolhida, por conveniência, a $12^{\mathrm{a}}$ sessão psicoterapêutica. A escolha ocorreu por essa sessão ter conteúdos terapêuticos significativos, fato esse justificado pelo TCM marcar, em quase toda a extensão da sessão, momentos de mudança - ciclos terapêuticos nos padrões de conversação entre paciente e psicoterapeuta, o que é necessário para o objetivo deste artigo, apresentar o instrumento.

$\mathrm{O}$ software $\mathrm{CM}$ deve estar previamente instalado em um computador. A sessão foi transcrita no programa bloco de notas do Windows 8.0, o qual é compatível com o software CM. Este apresenta um ícone em que deve ser anexada a sessão transcrita do bloco de notas. A partir desse procedimento, o software gerou o gráfico dos padrões de conversação da dupla paciente e psicoterapeuta. Com o gráfico gerado, voltou-se à transcrição da sessão a fim de se realizar uma análise exploratória do material clínico, bem como compreender a mudança demarcada por cada ciclo terapêutico, de forma a se identificar o conteúdo da conversação, pois o gráfico fornece tão somente o ponto específico da gravação da sessão em que fica demarcada a mudança (Khater \& Yoshida, 2011).

\section{Apresentação dos dados e discussão dos resultados}

Os gráficos gerados pelo software $\mathrm{CM}$ serão ilustrados a partir do caso Maria, diagnosticada com funcionamento borderline de personalidade. A avaliação prévia ao tratamento sugeriu a hipótese diagnóstica mencionada e indicou psicoterapia psicanalítica com 
a frequência de duas sessões semanais. A frequência indicada não foi possível devido a questões laborais e financeiras, e a paciente passou a ser atendida semanalmente no ambulatório de instituição parceira deste estudo (Silva et al.,2010). O tratamento durou nove meses e consistiu em 21 (vinte e uma) sessões, das quais a paciente compareceu a 13 (treze), sendo caracterizado por muitas faltas. O caso foi gravado em áudio e supervisionado semanalmente.

Visualiza-se, na Figura 4, o gráfico gerado pelo software CM referente a todo o processo psicoterapêutico de Maria.

$\mathrm{Na}$ Figura 4, pode-se observar que as sessões de 1 a 11 são caracterizadas pelo padrão de reflexão, em que a paciente manifesta processos reflexivos e cognitivos, refletindo sobre suas experiências, narrando sua história de vida. As sessões do início do processo de tratamento ( 1 a 6 ) demonstraram envolvimento da paciente no sentido de ela compartilhar com a psicoterapeuta sua história traumática e sofrida, realizando uma catarse. As intervenções da psicoterapeuta, em sua maioria, buscaram organizar a história de vida da paciente e também seu discurso. Nesse agrupamento de sessões, os padrões de tom emocional são reduzidos e os padrões de abstração são elevados.

Aos poucos, em torno da nona sessão, nota-se uma modificação no tom emocional, que passa a se elevar. As sessões intermediárias do processo psicoterapêutico (7 a 11) foram caracterizadas por faltas e atrasos em demasia - aspectos resistenciais e típicos do quadro diagnóstico da paciente. Nesse bloco de sessões, Maria compareceu às sessões 9 e 11, como é possível observar no gráfico. Portanto, o estilo narrativo presente nessas sessões ilustra que a paciente busca se esquivar de temas e experiências emocionalmente perturbadores que põem em risco seu equilíbrio interno, mostrandose resistente ao tratamento por meio de suas faltas.

A $12^{a}$ sessão ocorre em meio a uma sequência de presenças da paciente $(11,12,13$ e 14$)$, configurando importante marco no fluxo do trabalho psicoterapêutico. Apresenta o padrão de relaxamento, o que costuma ocorrer após a abordagem de temas sensíveis, permitindo ao paciente um momento de pausa para recuperar-se física e psiquicamente antes de dar

\section{Corpus Overview (custom Diditionaries)}

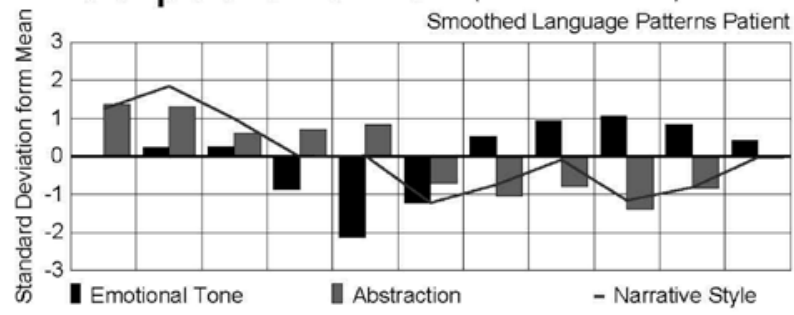

X Missing Data
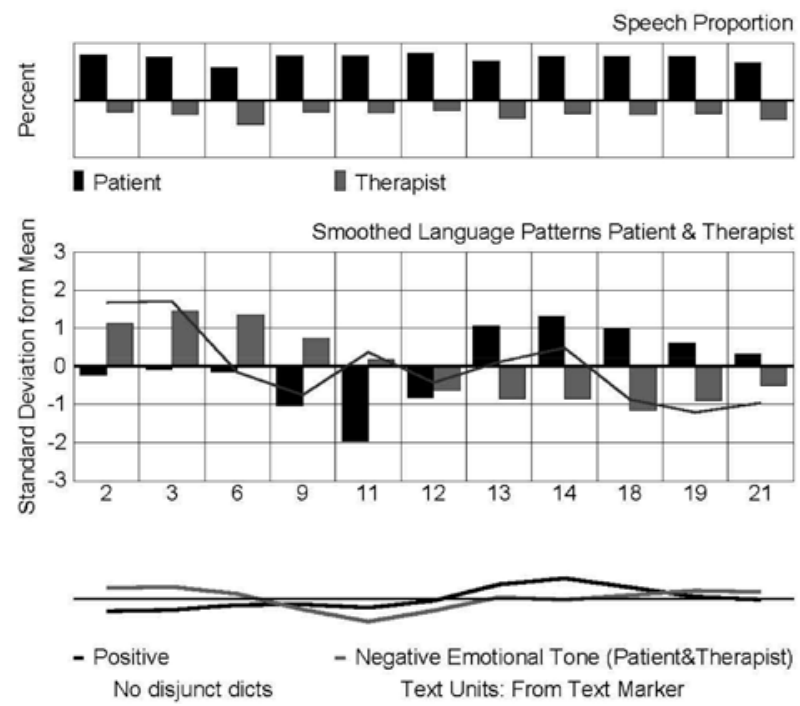

Verbalizações do paciente

e do psicoterapeuta:

utilizadas para ilustração

do TCM neste artigo

Figura 4. Gráfico de todas as sessões (corpus overview) do caso Maria pelo software CM. 
continuidade ao seu processo. Essa sessão é bastante ilustrativa do processo como um todo.

O padrão visível nas sessões seguintes (13 e 14) evidencia um processo elaborativo, em que a paciente se aproximou de maior compreensão acerca de si mesma e de seu sofrimento, desenvolvendo alguns insights com auxílio da psicoterapeuta. No gráfico, pode-se observar mudança de estilo, passando a se caracterizar pelo padrão de experiência. Neste, a paciente aborda tema conflituoso, sem ser capaz de elaborá-lo cognitivamente.

O final do processo (18 a 21), seguido de diversas faltas, culminou em um fechamento, que, apesar de precoce, pode ser considerado positivo em virtude do prognóstico esperado. Igualmente, a paciente conseguiu comparecer à sessão final, podendo despedir-se da psicoterapeuta e fazer um balanço das suas conquistas durante o tratamento.

A análise do processo psicoterapêutico completo pelo software CM (Figura 4) não identificou ciclos de mudança. Entretanto, o exame individual de cada sessão pelo instrumento permite a visualização de ciclos de mudança em todas as sessões. Assim, após caracterização geral do processo psicoterapêutico de Maria, foi selecionada a $12^{\mathrm{a}}$ sessão do caso para ilustrar a utilização do TCM como auxiliar na compreensão minuciosa de como se compôs esse trecho do processo, demonstrando o que pode ser realizado a partir do exame de cada sessão (Figura 5).

Pode-se observar, na Figura 5, nos padrões de conversação da paciente e da terapeuta, três círculos que representam ciclos de mudança, os quais demonstram momentos significativos da sessão de psicoterapia, vistos como clinicamente relevantes e associados à mudança. A análise qualitativa se dará apenas do primeiro ciclo, pois trata-se de uma ilustração do instrumento.

O início do primeiro ciclo é de reflexão (a barra emoção para baixo e a barra abstração para cima), ou seja, a paciente estava narrando sua rotina diária, os cursos que planejava fazer, entre outros assuntos. A seguir, pode-se observar que o padrão de abstração começa a diminuir e a emoção passa a se elevar. Quando ambos os padrões encontram-se elevados, o foco do discurso da paciente muda com o auxílio das intervenções da terapeuta: "Acho que dá uma vontade também, né, Maria, às vezes, de ser um pouco cuidada, né...”. As verbalizações de Maria passam a abranger seus sentimentos diante dos aspectos que vinha narrando de sua rotina, bem como o desejo em receber cuidados maternos, o que, por vezes, gera confusões nas suas relações com figuras femininas mais velhas.

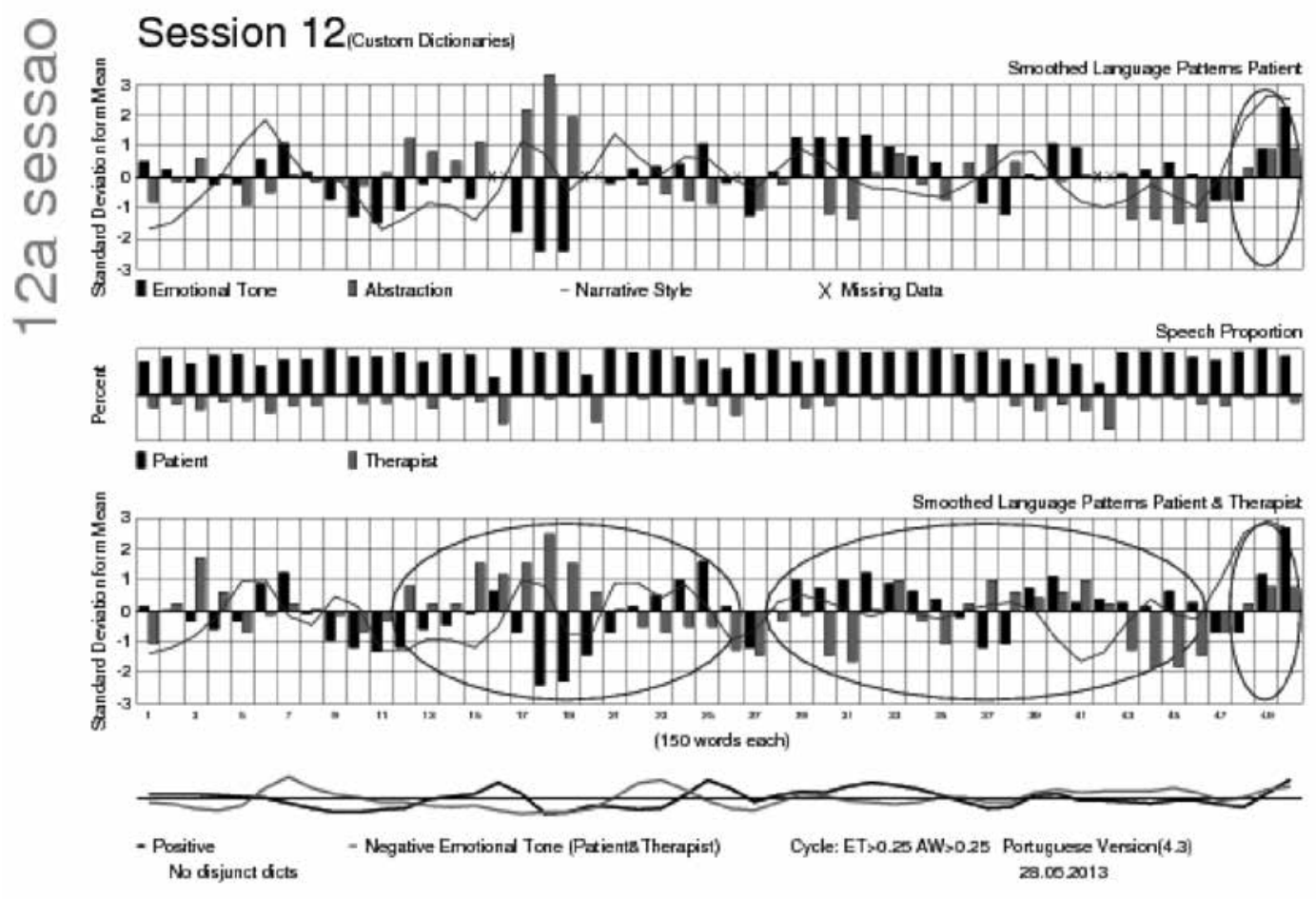

Figura 5. Gráfico da $12^{\mathrm{a}}$ sessão do caso Maria pelo software CM. 
Após período de conexão, inicia-se reflexão (emoção negativa e abstração positiva), momento no qual a paciente demonstra preocupação sobre decisão importante acerca de seu emprego. Posteriormente, o discurso de Maria começa a apresentar significativo nível emocional (padrão de experiência): "Tenho medo de ter alguma coisa na cabeça... de repente um tumor... sempre fui muito apavorada... tenho medo de morrer". A psicoterapeuta intervém: "Medo de morrer e de viver também, né”. Igualmente, a paciente retrata insegurança quanto às relações amorosas: "Ai, capaz que aquele vai gostar de mim[sic]". A paciente queixa-se, constantemente, de sua aparência, especialmente de seu sorriso, e de não ter recursos financeiros para satisfazer seus desejos de consumo. A intervenção da psicoterapeuta vai ao encontro de colocar em palavras o significado na queixa da paciente:

Psicoterapeuta: "... é interessante que tu falou do sorriso, mas a gente tava falando agora há pouco que é dificil de tu sorrir pra vida também, de tu gostar das coisas que tu tá fazendo, de tá satisfeita com as tuas escolhas, às vezes até de te sentir sem escolhas... [sic]" Paciente: "É... eu acho que eu nunca tive satisfeita com nada..." Psicoterapeuta: "E será que não é esse o sorriso que falta na tua vida? De poder sorrir pras tuas coisas, não só no que tu vê no espelho [sic]". Paciente: "Eu acho que eu olho e comeco a pensar o que eu fir até agora..."

É importante salientar que o TCM permite sustentar a ideia de que as mudanças da paciente são facilitadas pelas intervenções da psicoterapeuta, pois a análise exclusiva das verbalizações da paciente $\left(1^{\circ}\right.$ quadro da Figura 4) não apresenta ciclos de mudança e o quadro que expõe o padrão de interação paciente-psicoterapeuta ( ${ }^{\circ}$ quadro da Figura 4) apresenta três ciclos de mudança. Assim, essa ferramenta de análise do processo organiza o material proveniente de sessões de psicoterapia de forma a auxiliar a identificação dos momentos clinicamente significativos da interação entre paciente e terapeuta, corroborando com a ideia da psicoterapia como promotora de saúde.

\section{Considerações Finais}

A partir da apresentação do Modelo dos Ciclos Terapêuticos e do caso Maria, pode-se constatar que o instrumento pode ser um auxiliar de grande valia na compreensão do processo psicoterapêutico. Porém, ressalta-se a necessidade do olhar clínico e complementar, a fim de entender os processos latentes em conjunto com os manifestos na abordagem psicodinâmica/ psicanalítica de tratamento. O software possibilita ao usuário um norte para o estudo do processo, especialmente quando o objetivo é a investigação de processos longos de tratamento psicoterapêuticos como é o caso das psicoterapias psicodinâmica/psicanalítica. Assim sendo, ele funciona como um indicador e organizador de possíveis momentos associados à mudança a serem estudados em profundidade.

$\mathrm{Na}$ ilustração do caso, verifica-se a não ocorrência de ciclos terapêuticos no processo visto como um todo. Contudo, na análise individual de todas as sessões, foi possível identificar a ocorrência destes. Nesse sentido, destaca-se a necessidade de um estudo minucioso quando se pretende investigar as vicissitudes de um processo psicoterapêutico, pois, apenas olhar para o todo, não contempla a complexidade existente em cada sessão de um processo psicoterapêutico, excluindo a subjetividade intrínseca a ele. Considera-se que o fato de analisar apenas uma sessão como uma limitação na proposta deste artigo, mas espera-se que possa familiarizar possíveis novos usuários em sua utilização do TCM. Um estudo de caso detalhado, com o TCM aliado a análises clínicas de todas as sessões, auxiliará a compreensão do processo como um todo, bem como à seleção de aprofundamento de fragmentos significativos deste.

Ressalta-se que o instrumento apresenta limitações. Dentre elas, destaca-se possíveis regionalismos de linguagem no dicionário, que podem causar interpretações divergentes. Igualmente, alguns momentos vistos pelos pesquisadores como clinicamente significativos não foram contemplados pelo software, o que pode ser explicado pelo número reduzido de palavras inserido no dicionário de estilos narrativos até o momento, uma vez que são poucos os estudos com a língua portuguesa no Brasil. Além disso, os elementos não verbais (postura, comportamentos, gestos, ritmo e tonalidade da fala) e os acontecimentos entre sessões de tratamento, possivelmente envolvidos no processo de mudança, não são captados pelo software. Para tanto, seriam necessárias outras estratégias complementares na apreensão de processo tão complexo como o objeto de estudo em questão. Contudo, o TCM pode ser mais uma ferramenta no auxílio à prática psicoterapêutica do profissional.

\section{Referências}

Almeida, E. A. de (2010). Criação e aplicação de instrumento para verificação de aderência à técnica psicanalítica em 
sessões de psicoterapia (Dissertação de mestrado). Universidade Federal do Rio Grande do Sul, Programa de Pós-Graduação em Ciências Médicas: Psiquiatria, Porto Alegre.

Bucci, W. (2007). Pesquisa sobre processo. In E. S. Pearson, A. M. Cooper, \& G. O. Gabbard (Orgs.). Compêndio de Psicanálise (pp. 320-336). Porto Alegre: Artmed.

Brum, E. H. M. de, Frizzo, G. B., Gomes, A. L., Silva, M. da R., Souza, D. D. de, \& Piccinini, C. A. (2012). Evolução dos modelos de pesquisa em psicoterapia. Estudos de Psicologia (Campinas), 29(2), 259-269.

Buccheim, A., \& Mergenthaler, E. (2002). Adult attachment representation and computer-assisted language measures: What can we learn from the therapeutic cycle model for the adult attachment interview and vice versa? International Congress Series, 1241, 353-360.

Campezatto, P. v. M. (2014). Mudança psicológica no processo da psicoterapia psicanalítica. (Projeto de Doutorado não publicado). Pontifícia Universidade Católica do Rio Grande do Sul.

Cassel, P. A. (2014). Momentos de mudança em psicoterapia psicanalítica. (Dissertação de mestrado). Pontifícia Universidade Católica do Rio Grande do Sul.

Charman, D. (2003). Paradigms in current psychotherapy research: A critique and the case for evidence based psychodynamic psychotherapy research. Australian Psychologist, 38(1), 39-45.

Deakin, E. K., \& Nunes, M. L. T. (2008). Efetividade e eficácia na avaliação de resultados da psicoterapia psicanalítica com crianças. In M. N. Strey \& D. C. Tatim (Orgs.). Sobre ETs e Dinossauros: construindo ensaios temáticos (pp. 113-141). UFP: Passo Fundo, RS.

Eizirik, C. L. (2006). Psicanálise e pesquisa. Revista Brasileira de Psiquiatria, 28(3), 171-172.

Ferreira, E. B., Nunes, M. L. T., Kurth, R. A., Pokorny, D., Terra, L., Hauck, S., \& Ceitlin, L. H. F. (2006). Desenvolvimento da versão em português do Brasil do questionário sobre padrão de relacionamentos Beziehungs-Muster Fragebogen. Revista de Psiquiatria do Rio Grande do Sul, 28(3), 276-288.

Ferreira, S. N., \& Yoshida, E. M. P. (2004). Produção científica sobre psicoterapias breves no Brasil e demais países latino-americanos (1990-2000). Estudos de Psicologia, 9(3), 523-531.
Fishman, D. (2002). From single case to database: A new method for enhancing psychotherapy, forensic, and other psychological practice. Applied \& Preventive Psychology, 10(4), 275-304.

Fontao, M., \& Mergenthaler, E. (2002). Das therapeutische zyklusmodell: Eine evaluation im gruppenpsychotherapeutischen setting. Gruppen psychotherapie und Gruppen dynamik, 38, 349-371.

Gelo, O., \& Mergenthaler, E. (2003). Psicoterapia e linguaggio metaforico. Psicoterapia, 27, 53-65.

Greenberg, L. S., \& Newman, F. L. (1996). An approach to psychotherapy change process research: Introduction to the special section. Journal of consulting and clinical psychology, 64(3), 435-438.

Hauck, S., Crestana, T., Mombach, C. K., Almeida, E. A. de, \& Eizirik, C. L. (2008). Pesquisa em psicanálise e psicoterapia psicanalítica: Um novo instrumento para avaliação de aderência à técnica em estudos de efetividade. Revista Brasileira de Psiquiatria, 30(3), 290-301.

Kächele, H., Thomä, H., Ruberg, W., \& Grünzig, H.J. (1988). Audio-recordings of the psychoanalitic dialogue: Scientific, clinical and ethical problems. In Dahl, H., Kächele, H., \& Thomä, H. (Orgs.), Psychoanalytic Process Research Strategies (pp. 179-193). Berlin/Heidelberg/New York: Springer.

Kächele, H. (2000). Conventional wisdom and/or evidence based psychotherapy. In S. Gril, A. Ilbanez, I. Mosca \& P. Souza (Orgs.), Investigación en psicoterápica: processos y resultados (pp. 17-26). Pelotas: Educat.

Khater, E., \& Yoshida, E. M. P. (2011). Desenvolvimento de um dicionário personalizado do Modelos dos Ciclos Terapêuticos (TCM) para adolescentes em conflito com a lei. Psicologia: Teoria e Prática (Impresso), 13, 168-181.

Krause, M., de la Parra, G., Arıstegui, R., Dagnino, P., Tomicic, A., Valdés, N., Echávarri, O., Vilches, O., Ben-Dov, P., Strasser, K., Reyes, L., Altimir, C., \& Ramírez, I. (2007). The evolution of therapeutic change studied through generic change indicators. Psychotherapy Research, 17, 673-689.

Lepper, G., \& Mergenthaler, E. (2005). Exploring group process. Psychotherapy Research, 15(4), 433-444.

Levitt, H., Butler, M., \& Hill, T. (2005). What clients find helpful in psychotherapy: Developing principles 
for facilitating moment-to-moment change. Journal of Counseling Psychology, 53(3), 314-324.

Marcolino, J. A. M., \& Iacoponi, E. (2001). Escala de aliança psicoterápica da Califórnia na versão do paciente. Revista Brasileira de Psiquiatria, 23(2), 88-95.

Mergenthaler, E. (1996a). Text analysis and computers. Germany: Mannheim, ZUMA. ISBN 3-924220-11-5.

Mergenthaler, E. (1996b). Emotion-abstraction patterns in verbatim protocols: A new way of describing psychotherapeutic processes. Journal of Consulting and Clinical Psychology, 64(60), 1306-1315.

Mergenthaler, E. (2008). Resonating minds: A schoolindependent theoretical conception and its empirical application to psychotherapeutic processes. Psychotherapy Research, 18(2), 109-26.

Mergenthaler, E., \& Bucci, W. (1999) Linking verbal and non-verbal representations: Computer analysis of referential activity. British Journal of Medical Psychology, 72(3), 339-354.

Meyer, A. E. (1988). What makes psychoanalysts ticks? A model and the method of audio-recorded retroreports. In Dahl, H., Kächele, H., \& Thomä, H. (Orgs.). Psychoanalytic Process Research Strategies (pp. 272-290). Berlin/Heidelberg/New York: Springer.

Nunes, M. L. T. (1995). O uso do gravador para pesquisa em psicoterapia. Psico, 26(2), 121-132.

Peuker, A. C., Habigzang, L. F., Koller, S. H., \& Araújo, L. B. (2009). Avaliação de processo e resultado em psicoterapias: Uma revisão. Psicologia em Estudos (Maringá), 14(3), 439-445.

Sanchez, L. F. (2014). Momentos associados à mudança em psicoterapia psicanalitica de adultos. (Dissertação de
Mestrado). Pontifícia Universidade Católica do Rio Grande do Sul.

Serralta, F. B., Nunes, M. L. T., \& Eizirik, C. L. (2007). Elaboração da versão em português do Psychotherapy Process Q-Set. Revista de Psiquiatria do Rio Grande do Sul, 29(1), 44-55.

Serralta, F. B., Nunes, M. L. T., \& Eizirik, C. L. (2011). Considerações metodológicas sobre o estudo de caso na pesquisa em psicoterapia. Estudos de Psicologia (Campinas), 28(4), 501-510.

Serralta, F. B., \& Streb, L. G. (2003). Notas sobre pesquisa em psicoterapia psicanalítica: Situação atual e perspectivas. Revista Brasileira de Psicoterapia, 5(1), 53-65.

Silva, M. R., Halberg, A. E., Steibel, D., \& Campezatto, P. V. M. (2010). A resistência no início do tratamento estudo do processo da psicoterapia psicanalítica. (Projeto de pesquisa não publicado).

Yoshida, E. M. P. (2008). Modelo dos ciclos terapêuticos: Dicionários em português e estudos de validade. (Projeto de Pesquisa). Campinas: Pontifícia Universidade Católica de Campinas. Manuscrito.

Yoshida, E. M. P., \& Mergenthaler, E. (2011). Coincidência de conexão do tom emocional com abstração e o resultado psicoterapêutico. Estudos de Psicologia (Campinas), 28(1), 35-43.

Yoshida, E. M. P., \& Silva, F. R. C. S. (2007). Escala de avaliação de sintomas-40 (EAS-40): Validade e precisão em amostra não clínica. Psicologia Escolar e Educacional, 11(1), 89-99.

Recebido em: 08/05/2014

Primeira Reformulação em: 18/08/2014

Aprovado em: 11/12/2014 
Sobre os autores:

Paula Argemi Cassel é psicóloga, mestre em Psicologia Clínica, doutoranda em Cognição Humana - PUCRS, e bolsista CAPES.

Lívia Fração Sanchez é psicóloga, mestre em Psicologia Clínica - PUCRS, e sócia graduada do IEPP.

Paula von Megden Campezatto é psicóloga, psicoterapeuta de crianças, adolescentes e adultos; mestre e doutoranda em Psicologia Clínica - PUCRS, bolsista do CNPq e sócia efetiva do IEPP.

Eduardo Khater é psicólogo, doutor em Psicologia pela PUC-Campinas e membro da Society for Psychotherapy Research.

Maria Lúcia Tiellet Nunes é psicóloga, doutora em Psicologia Clínica pela Universidade Livre de Berlim e professora titular aposentada da PUCRS.

Elisa Yoshida é psicóloga, pós-doutora pela Université de Montréal, Canadá.

Contato com os autores:

Paula Argemi Cassel

Avenida Ipiranga, 6681. Partenon.

Porto Alegre/RS

CEP: 90619-900.

E-mail: paula.acassel@gmail.com 